\title{
CONFORMA: A PROGRAM FOR DETERMINING RING CONFORMATIONS AND PUCKERING COORDINATES
}

\author{
J. Iulek ${ }^{*}$ \\ Instituto de Química de São Carlos - USP - São Carlos - SP \\ J. Zukerman-Schpector ${ }^{\#}$ \\ Departamento de Química - Universidade Federal de São Carlos - CP 676 - 13565-905 - São Carlos - SP
}

Recebido em 15/5/96; aceito em 12/12/96

\begin{abstract}
CONFORMA: A PROGRAM FOR DETERMINING RING CONFORMATIONS AND PUCKERING COORDINATES. A program (CONFORMA) was developed to calculate ring puckering coordinates using Cremer and Pople's (J. Am. Chem. Soc. 1975, 97, 1354) methodology, which quantifies the conformation of five or more atom rings. The program performs, also, a conformational analysis based on these results.
\end{abstract}

Keywords: ring conformation; puckering coordinates.

\section{INTRODUCTION}

Many research areas require precise determination of the conformations assumed by different atom rings, as for example the study of interactions protein-substrate or crystal structure determinations of chemicals. Therefore, a methodology for quantifying the different conformations, allowing an easy and fast comparation between them, is necessary. This kind of problem becomes more complex when one needs to identify or compare hybrid conformations. Several methodologies have been proposed $^{1,2,3}$, all aiming at systematizing and quantifying the ring conformations. Among them, Cremer \& Pople's ${ }^{4,5}$, which independs on the number and type of atoms, is the most widely used, this methodology defines a set of ring puckering coordinates, their number depending on the number of atoms in the ring, which describe the nonplanar character of a ring in a unique way. CONFORMA is a program developed for computers with the MS-DOS operational system, it calculates the puckering coordinates, for five or six member rings as defined by Cremer $\&$ Pople, with their standard deviations according to Norrestam ${ }^{6}$, and the conformation the rings assume. All calculations are performed using atomic coordinates from crystal structure determination or from any other cartesian coordinate system.

\section{PROGRAM DESCRIPTION}

For five member rings, CONFORMA calculates the $\mathrm{q}_{2}$ and $\phi_{2}$ coordinates, which are related to the departure from planarity and to the position in the pseudo-rotation pathway, respectively. For six member rings, three coordinates, $\mathrm{q}_{2}, \mathrm{q}_{3}, \phi_{2}$ are calculated, and, as described by Cremer \& Pople ${ }^{4}$, also the corresponding spherical polar coordinates $(\mathrm{Q}, \theta, \phi)$, which locate the various possible ring conformations on the surface of a sphere. Thus, a perfect chair has $\theta$ equal to 0 or $180^{\circ} ; \theta$ equal to $90^{\circ}$ corresponds to a boat or twisted boat conformation, with $\phi$ defining the position of the pseudo-rotation pathway. In the case of hybrid conformations, this will be reflected in the values of $\theta$ and $\phi$. Q is related to the departure from planarity and is therefore called total puckering amplitude.

\footnotetext{
* Permanent address: Departamento de Química - Universidade Estadual de Ponta Grossa - Campus Universitário de Uvaranas 84031-510 - Ponta Grossa - PR

E-mail: iulek@ifqsc.sc.usp.br

\# E-mail: julio@power.ufscar.br
}

Finally, the program performs an analysis and interprets all these puckering coordinates.

\section{EXAMPLES}

Two crystal structures will be shown as examples. In figure 17 ring $\mathrm{A}$ is in a slightly distorted chair conformation, and in compound of figure $3^{8}$ the four rings are examinated, special atention should be given to ring $\mathrm{B}$ which is a highly strained six member ring, whose conformation is not easy to evaluate visually, but puckering coordinates make this evaluation straightforward.

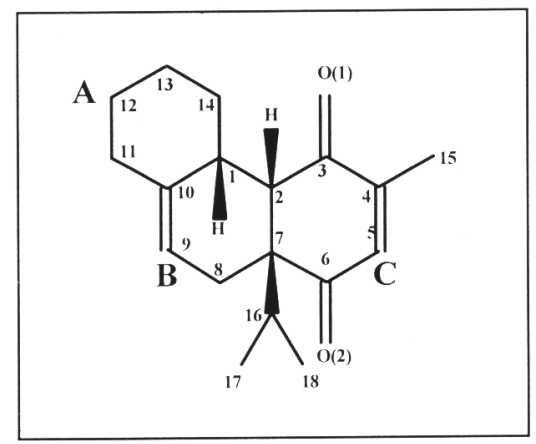

Figure 1. Atom numbering and ring letter designation scheme of 7-isopropyl-4-methyl-tricyclo[8.4.0.0 $\left.0^{2,7}\right]-4,9$-tetradecadiedone-3,6.

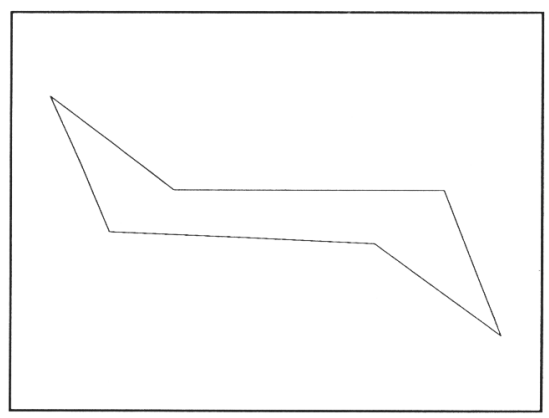

Figure 2. Projection of Ring A from figure 1.

The Puckering Coordinates are: $\mathrm{q}_{2}=0.042(6) \AA, \mathrm{q}_{3}=0.568(6) \AA, \mathrm{Q}$ $=0.570(6) \AA, \theta=4.2(6)^{\circ}$ and $\phi=306(8)^{\circ}$;

Approximated percentual contributions for Ring A conformation: Pure Chair $(C)=90.7 \%$, Half-Boat $(H B)=7.4 \%$, Half-Chair $(H C)=1.9 \%$ 


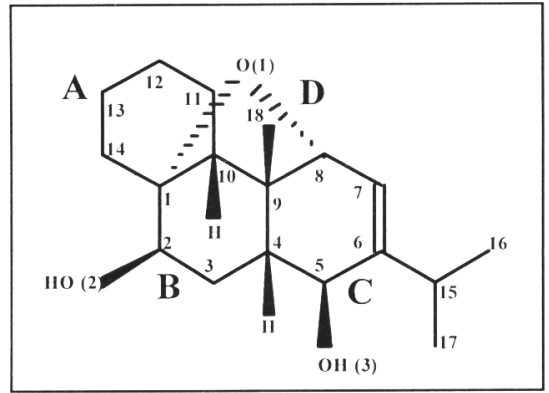

Figure 3. Atom numbering and ring letter designation scheme of 2,5-dihydroxy-6-isopropyl-9-methyl-1,8-oxy-tricyclo [8.4.0.0 $\left.0^{4,9}\right]$ -6-tetradecene.

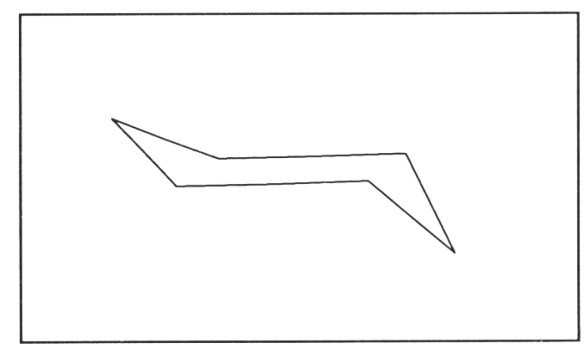

Figure 4. Projection of Ring A from fig. 3

The Puckering Coordinates are: $q_{2}=0.167(4) \AA ; q_{3}=-0.489(4) \AA$; $Q=0.517(4) \AA ; \theta=161.1(5)^{\circ} ; \phi=2(1)^{\circ}$.

Approximated percentual contributions for Ring A conformation: Pure Chair $(C)=58.0 \%$; Half-Boat $(H B)=38.8 \%$; Half-Chair $(\mathrm{HC})=3.2 \%$.

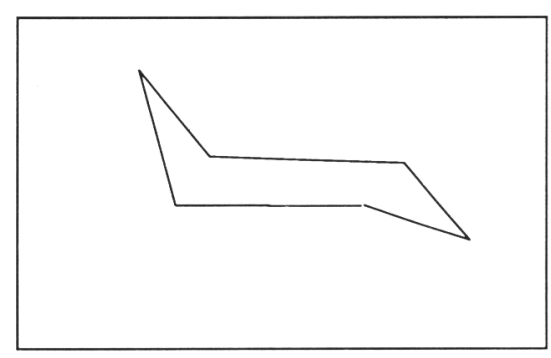

Figure 5. Projection of Ring $\boldsymbol{B}$ from fig. 3

The Puckering Coordinates are: $q_{2}=0.302(4) \AA ; q_{3}=-0.569(4) \AA$; $Q=0.644(4) \AA ; \theta=152.0(3)^{\circ} ; \phi=127.0(7)^{\circ}$.

Approximated percentual contributions for Ring $\boldsymbol{B}$ conformation: Half-Boat $(H B)=47.7 \%$; Pure Chair $(C)=37.8 \%$; Half-Chair $(\mathrm{HC})$ $=14.5 \%$.

\section{CONCLUSION}

In future versions of the program we intend to implement a graphical facility that will allow the visualization of the departure of hybrid conformated rings from its corresponding pure conformations.

Free copies of the program may be requested from the authors, by letter or e-mail. The program will be sent in a 3.5" diskette for IBM-PC and compatibles, which contains the following files: readme.1st, with starting information;

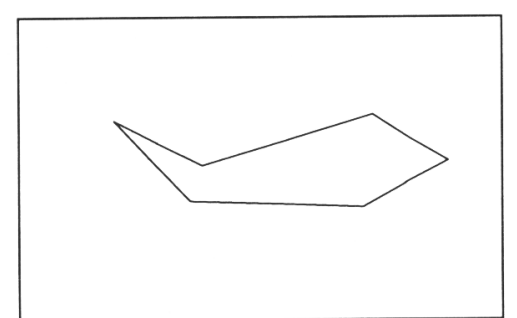

Figure 6. Projection of Ring $C$ from fig. 3

The Puckering Coordinates are: $q_{2}=0.413(4) \AA ; q_{3}=-0.289(4) \AA$; $Q=0.504(4) \AA ; \theta=125.0(4)^{\circ} ; \phi=157.6(5)^{\circ}$

Approximated percentual contributions for

Ring C conformation: Half-Chair $(H C)=58.1 \%$; Half-Boat $(H B)=$ $19.7 \%$; Twisted Boat $(T B)=16.6 \%$; Pure Boat $(\mathrm{B})=5.6 \%$.

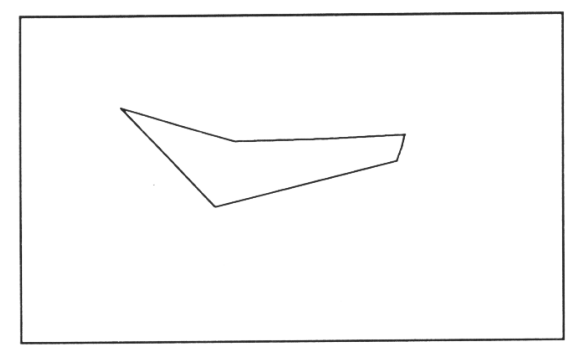

Figure 7. Projection of Ring $\boldsymbol{D}$ from fig. 3

The Puckering Coordinates are: $q_{2}=0.437(4) \AA ; \phi_{2}=82.0(4)^{\circ}$. Approximated percentual contributions for Ring $\boldsymbol{D}$ conformation: Pure Twist $(T)=55.6 \%$; Pure Envelope $(E)=44.4 \%$.

userregi.doc, the user registration form, which the authors kindly ask users to fill and return; id.coo, which identifies the coordinate files/examples, included in the package; [name].dat, example files with the appropriate format for CONFORMA; CONFORMA.EXE, the executable version of CONFORMA program.

\section{ACKNOWLEDGMENTS}

This work has received partial support from CNPq, FAPESP and CAPES.

\section{REFERENCES AND NOTES}

1. Dunitz, J. D.; Waser, J.; J. Am. Chem. Soc. 1972, 94, 5645.

2. Altona, C.; Sundaralingam, M.; J. Am. Chem. Soc. 1972, 94,8205

3. Dunitz, J. D.; Tetrahedron 1972, 28, 5459.

4. Cremer, D.; Pople, J. A.; J. Am. Chem. Soc. 1975, 97, 1354.

5. A program, RING (Cremer, D. QCPE, 1975, 11, 288) written in FORTRAN IV (CDC), only calculates de puckering coordinates.

6. Norrestam, R.; Acta Cryst. 1981, A37, 764.

7. Zukerman-Schpector, J.; Iulek, J.; Silva Jr., F.; Brocksom, T. J.; Acta Cryst. 1994, C50, 85.

8. Zukerman-Schpector, J.; Iulek, J.; Silva Jr., F.; Brocksom, T. J.; Acta Cryst. C. 1997, no prelo. 